\title{
"Know your CD4 campaign": 6-year outcomes from a quality improvement initiative to promote earlier initiation of antiretroviral therapy in Tanzania
}

Peter Memiah, Constance Shumba1, Yvonne Henley, Sekela Mwakyusa ${ }^{2}$, Abuu Maghimbi', Patience Komba ${ }^{2}$, Anthony Mlila ${ }^{2}$, Venosa Haule ${ }^{2}$, Tuhuma Tulli ${ }^{2}$, Stafford Kristen, Martine Etienne-Mesubi, Carla Alexander

School of Medicine, Institute of Human Virology, University of Maryland, Baltimore, MD, USA, ${ }^{1}$ Kampala, Uganda, ${ }^{2}$ Dar-esSalaam, Tanzania

Address for the Correspondence: Dr. Peter Memiah, School of Medicine, University of Maryland, Baltimore 21212, USA.

E-mail: pmemiah@gmail.com

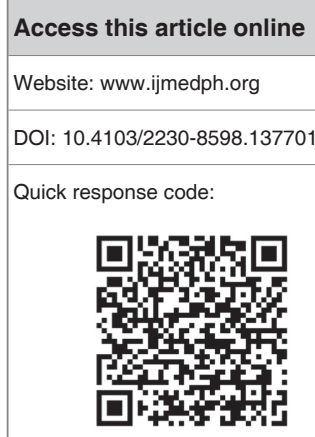

Background: Late initiation of treatment for illness secondary to the human immunodeficiency virus (HIV) remains a major challenge in developing countries. Despite the World Health Organization (WHO) recommendation that treatment be initiated early in disease management, health providers conducting quality improvement monitoring in one region of Tanzania noted that common management practice relies upon clinical signs of advanced disease alone for initiation of combination antiretroviral therapy (ART). Although Tanzanian National Treatment Guidelines followed standard WHO recommendations, few patients initiated ART based on laboratory parameters. As a potential barrier to optimal patient outcomes, further investigation of this inconsistency led to recognition of challenges reflecting patient, healthcare staff, and laboratory levels that might inhibit the use of CD4 cell counts as the entryway to care. Materials and Methods: Using a quality improvement approach, investigations were pursued for six discrete activities of HIV care delivery with before and after measures of selected indicators. With respect to patient engagement, meetings and informal educational sessions were held to promote understanding of the meaning of and need for CD4 testing. For clinic staff: (1) Qualitative interviews were conducted with providers to understand why laboratory data was not being used and (2) on-site interviews were conducted with laboratory personnel to review beliefs, methods, and practices related to measurement of CD4 cells testing. A large scale local campaign was mounted to (1) educate and empower patients to recognize a need for CD4 information in management of their own care; (2) re-educate and encourage providers to use measured, rather than clinical observation alone to initiate ART; and (3) understand and resolve clinical and laboratory challenges. Based upon findings from the interviews: (1) Meetings with hospital administrations were effected to resolve institutional barriers to using CD4 cell testing. Specific on-site training was initiated for both providers, with regard to use of CD4 cell counts, and nurses, with advanced training to initiate routine CD4 testing. These activities were well received because all staff were able to review unlinked, site-based clinical data to appreciate gaps in a local care. Results: The number of CD4 samples obtained and recorded increased by $114 \%$ between May and October 2007 at targeted health facilities. ART enrollment increased by $62 \%$ between June and September 2007 without other significant change in care delivery. The median baseline CD4 at enrollment increased from 110 cells $/ \mathrm{mm}^{3}$ in June to 150 cells $/ \mathrm{mm}^{3}$ in September. Overall retention rate was $77 \%$ for 13,333 HIV patients enrolled in seven facilities. In September 2013, the cumulative 6-year overall retention rates are $77 \%$ for 53,040 patients enrolled in 42 health facilities in the region. Obstacles were addressed and community empowerment techniques used to stimulate change in established clinical behaviors. Conclusion: This "Know your CD4 campaign" initiative resulted in increased uptake of CD4 testing, treatment initiation and an unanticipated improvement in patient retention. With attention to patient, staff, and laboratory elements in resource-poor settings, decline in immune function and morbidity may be reduced and viral suppression prolonged. Empowering patients to be involved in their own care resulted in better overall adherence with HIV management. Local use of reviewed data can impact overall effectiveness of HIV care delivery. Simple quality improvement approaches impact sustainable change.

Key words: Antiretroviral therapy, community empowerment, human immunodeficiency virus/AIDS, quality improvement 


\section{INTRODUCTION}

Antiretroviral therapy (ART) has had a dramatic impact in mortality rates among those living with HIV in both, developed and nondeveloped world ${ }^{[1]}$ with comparable rates of adherence and viral suppression. However, individuals continue to die to as a result of late presentation ${ }^{[2,3]}$ and delayed uptake of ART. ${ }^{[4]}$ The introduction of combination ART has dramatically improved clinical outcomes in patients with HIV infection even in resource-limited settings. The impact of these treatments on reducing mortality has transformed HIV/AIDS from a rapidly progressing terminal disease to a chronic condition. ${ }^{[1,5-8]}$ The effectiveness of HIV treatment is impacted by many factors including:

a. Immune status of the patient at the time of treatment initiation for example, CD 4 counts $<100$ cells $/ \mathrm{mm}$ have poor outcomes

b. Co-morbid infections and inadequate management options for example resistant tuberculosis (TB), cryptococcal meningitis, or recurrent bacterial infections ${ }^{[0]}$

c. Previous treatment history including interruptions in HIV treatment ${ }^{[8]}$ and

d. Limited laboratory facilities for monitoring disease activity and safety of antiretroviral treatment.

In resource-constrained settings, lack of resilient infrastructure plays a role in inhibiting access to early initiation of therapy. As countries escalate effective HIV/AIDS programs, weaknesses in national health systems have become apparent. Sub-Saharan Africa, suffering the greatest burden of the HIV epidemic, ${ }^{[10,11]}$ has resulted in challenges to workforce capacity and other human resource issues. ${ }^{[12]}$ The scaling up of ART ${ }^{[13,14]}$ and voluntary HIV counseling and testing (VCT) in combination with earlier ART, ${ }^{[15]}$ and monitoring of care delivery and monitoring systemic improvements, ${ }^{[16]}$ have shown promising results. Regardless of steps forward, enormous and diverse challenges, such as late ART initiation and high rates of loss to follow-up, still persist. ${ }^{[17-19]}$

The rapid scale up of ART as a result of the introduction of the US President's Emergency Plan for AIDS Relief and the WHO's 3 by 5 initiative in 2004 required a collaborative approach to the delivery of HIV/AIDS services. The United Republic of Tanzania in East Africa, one of 15 target countries initially, used a regionalization model for coordinating implementing partners (IP) to combat the HIV/AIDS epidemic. One IP was a consortium led by Catholic Relief Services with technical assistance provided by the University of Maryland, School of Medicine - Institute of Human Virology, Futures Group International, Interchurch Medical Assistance - World Health, and Catholic Medical Mission Board (not active in Tanzania). Working in four regions to assist the Regional Health Medical Teams (RHMT) in implementing effective care and treatment for persons living with HIV/AIDS, the IP followed Tanzania National Guidelines based upon WHO recommendations for disease management.

Challenges exist at each step of implementation of HIV care and treatment from timely HIV diagnosis through enrollment in care, staging and determination of eligibility for ART, initiation of ART, and on-going adherence and retention in care. ${ }^{[19]}$ As healthcare moves away from a focus on episodic, acute care toward more holistic, continuous care, there is an embedded acknowledgement of the need to ensure smooth transitions between health, social, community and other stakeholders. ${ }^{[20]}$ This move toward integration results in a need for novel solutions that can improve the efficiency and effectiveness of the existing workforce, while providing sustainable quality and efficiency in care delivery. ${ }^{[21]}$ Use of a multidisciplinary care strategy with different health cadres and professionals focused upon a common purpose is well-documented in other health delivery settings.

This article describes an innovative quality improvement project dubbed the "Know your CD4 campaign" that included multiple reinforcing activities: specifically, increasing patient ownership with an emphasis on participation in their own healthcare; staff education and training emphasizing national guidelines that require CD4 cell counts to trigger antiretroviral use; and laboratory testing and staging of disease that improve clinical systems and care. Sustainable retention patterns have been maintained in this region despite rapid scale up that followed this campaign.

\section{MATERIALS AND METHODS}

This before and after design, "Know your CD4 campaign," was a clinical quality improvement and educational intervention to promote earlier initiation of ART for persons living with HIV in one rural region in Tanzania. Activities were carried out between July 2007 and October 2007 in one region of Tanzania. Activities began at the Regional Hospital and were cascaded to six-district level hospitals government operated. Through focus group discussion, health care providers described being unable to rely upon laboratory results for initiation of ART with unreliable equipment and frequent delays in obtaining reagents. Barriers to early enrollment on ART were identified using a "root cause" analysis and multidisciplinary technical assistance teams conferred with hospital administration, providers and patients extending the same practices to District Hospitals.

The "Know your CD4 campaign" consisted of a multidisciplinary approach to address the following challenges at these different levels.

\section{At the clinic level}

- At the point of triage for the clinic, if no CD4 cell count was recorded in the chart, the patient was sent to the laboratory for this testing

- Incentives for patients to learn their own CD4 cell count were used such as pens and promotional t-shirts for those who enrolled as "CD4 ambassadors" (peer educators) for HIV patients in the community

- The CD4 ambassadors were trained to assume selected nonclinical responsibilities from service providers such as conducting health education sessions at the clinic along with clinic staff and at the community level 
- A buddy system emphasizing self-care behaviors was instituted such as methods of disclosure, understanding CD4 quantification and adherence to ART

- Health care providers were re-educated on the importance of CD4 cell counts

- Pharmacies ensured unexpired and adequate drugs on-site to treat newly identified people.

\section{At the community level}

- Distribution of brochures at stand-alone VCT and TB centers emphasizing the importance of immediate referral of HIV-positive patients to a clinic for follow-up and evaluation

- Community mobilization efforts through existing support groups included sensitization campaigns with a focus on stigma reduction and the importance of disclosure; a simplified understanding of the meaning of CD4 and encouragement to request testing.

\section{At the laboratory level}

- At the laboratory an inventory of equipment; revised laboratory policies and procedures including joint meetings between laboratory personnel and administration to educate them about procedures

- Co-ordination meetings at each hospital and among all hospitals took place at which the daily capacity of the CD4-counting machine was discussed, the number of samples per site was quantified, and planning for the best utilization of available laboratory capacity and sample transportation was discussed, with the allocation of specific days on which particular facilities would send samples

- Better communication with the laboratories and flagging of patient files alerted health care providers to the availability of CD4 results.

There were 17 members of the multi-disciplinary team (nurses, adherence support, and pharmacy (two); laboratory workers (two); doctors (five); managerial staff (four); and community-based health workers (four).

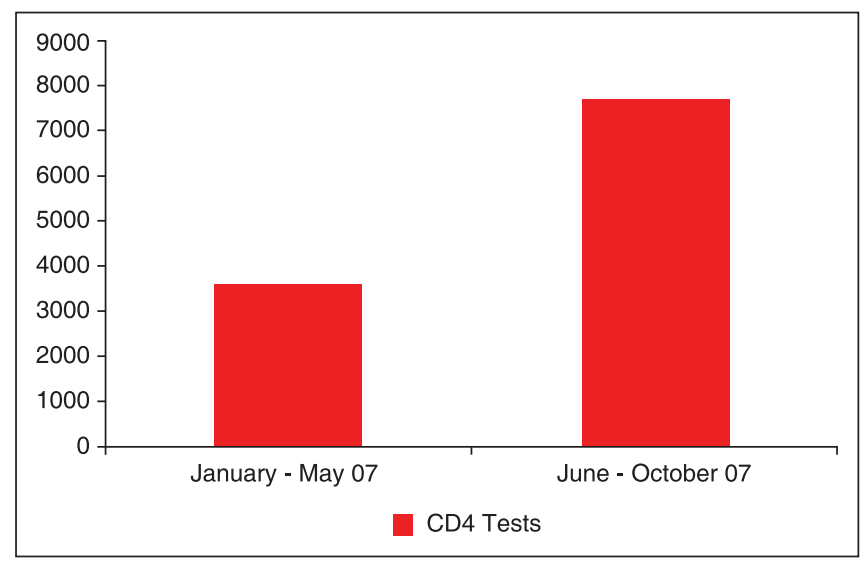

Figure 1: Number of CD4 count tests
The primary objectives of this "Know your CD4 campaign" was to improve clinical systems and care; to improve staff education and training (emphasizing national guidelines that require CD4 counts, laboratory testing, and staging), and to increase patient ownership with an emphasis on participation in their own healthcare.

Baseline data were collected at the participating facilities in May 2007. Postintervention data for different measures was collected at specific time points in September 2007, February 2008 and December 2012 to ensure there were sustainable gains to this intervention.

\section{Ethical considerations}

Permission was obtained from the Tanzanian RHMT and the Council Health Management Teams (CHMT) before embarking on this "Know your CD4 campaign." This quality improvement intervention was a highly participatory approach with the RHMT and CHMT and hospital administration teams taking the lead. All data were collected and stored in an aggregate format without patient identifiers. The data were confidential and anonymous. Results of the quality improvement project were summarized and communicated with regional authorities to improve local care delivery.

\section{RESULTS}

The number of CD4 cell count samples increased by $114 \%$ from May (3585 samples) to October 2007 (7698 samples) [Figure 1].

Test results prior to the "Know your CD4 campaign" took 2-3 days, whereas during intervention and thereafter results were being received on the same day. For facilities that had no laboratory equipment proper networking was done to ensure efficient test results were received in less than a day. Median CD4 increased from 110 cells $/ \mathrm{mm}^{3}(n=122)$ to 158 cells $/ \mathrm{mm}^{3}(n=658)$ [Figure 2].

Patient retention increased from 69\% in December 2007 to $72 \%$ in February 2008. ART enrollment increased by $62 \%$ between June and September 2007 [Figure 3].

Overall retention at the end of December 2007 was at $77 \%$ for 13,333 HIV patients enrolled in these seven facilities. Despite the scale up of ART efforts to other facilities in the region, retention was maintained at $77 \%$ as at September 2013 for 42 health facilities who had enrolled 53,040 HIV patients [Figure 4].

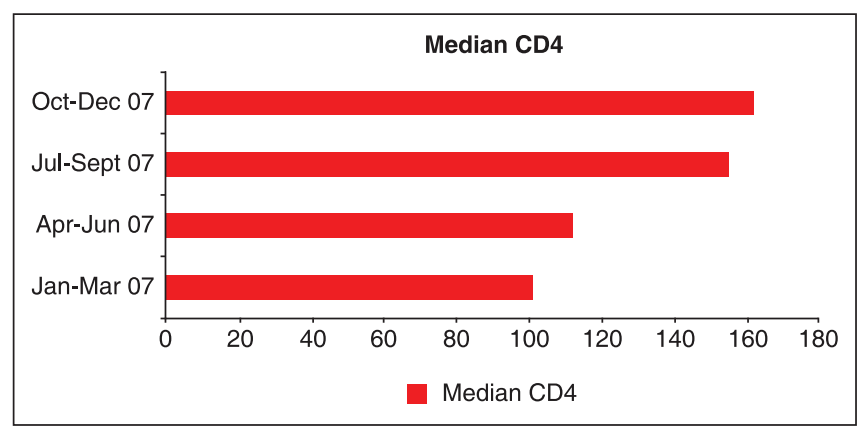

Figure 2: Median CD4 counts 


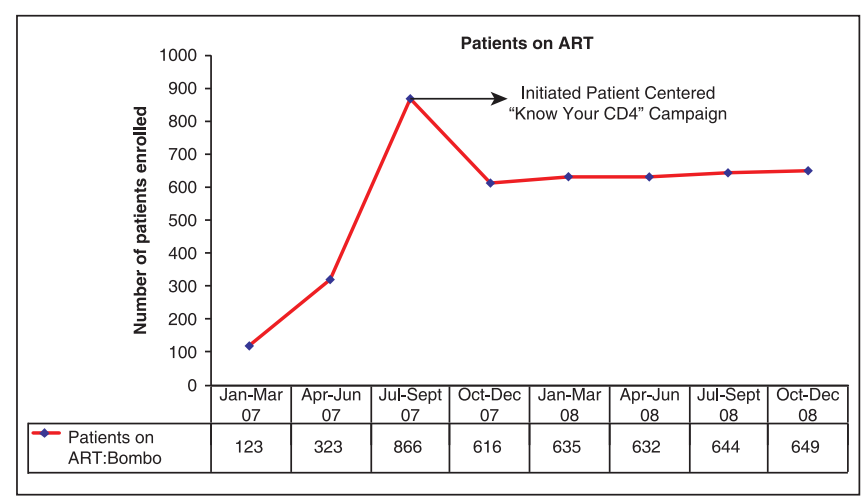

Figure 3: Number of patients on antiretroviral therapy over time

\section{DISCUSSION}

The "Know your CD4 campaign" a quality improvement initiative in rural Tanzania resulted in health care providers engaging in early initiation of therapy. The multi-disciplinary quality improvement team identified lack of knowledge about CD4 cell counts and lack of testing by clinicians as a barrier to timely initiation of treatment, and developed an intervention to improve the use of CD4 counts in clinical decision-making. This process also raised awareness of the value of knowing one's CD4 count among patients who became more involved and knowledgeable regarding their own care.

High HIV viral measurements and low CD4 cell counts are independently associated with mortality. ${ }^{[2]}$ In a recent randomized trial conducted in Thailand among 713 ART naïve patients, showed that using CD4 counts alone to monitor highly active ART in HIV treatment programs in resource-limited settings is an appropriate strategy to use as viral load measurement becomes more affordable and feasible in these settings. ${ }^{[23]}$ Monitoring of the HIV viral measurement is central to therapeutic HIV management worldwide and recommended in the WHO 2013 consolidated guidelines. ${ }^{[24,25]}$ However, in low- and middle-income countries, with limited resources and restricted access to costly second- and third-line drugs, the utility of this approach continues to be debated. ${ }^{[26,27]}$ Without laboratory monitoring, patients may experience prolonged virologic failure and resultant mutations signifying drug resistance. This limits second-line treatment options, increases morbidity, mortality and increases transmission of resistant viruses in the population..$^{28,29]}$

Significant resources have been applied toward laboratory-strengthening programs in resource-limited settings. Reliable laboratory services are critical for meeting the health-related Millennium Development Goals of the United Nations. ${ }^{[30]}$ Quality-assured laboratory support is critical for any HIV/AIDS treatment, care, and prevention programs because patients are accurately diagnosed and treated for HIV, as well as other opportunistic infections such as TB, and monitors the progress of ART, patient adherence, and quality of care. Within the laboratories, equipment was used to maximum capacity, specimen handling improved and the number of specimens from each site increased. CD4 campaign improved cooperation among laboratory staff from different sites also improved. With the inception of facility

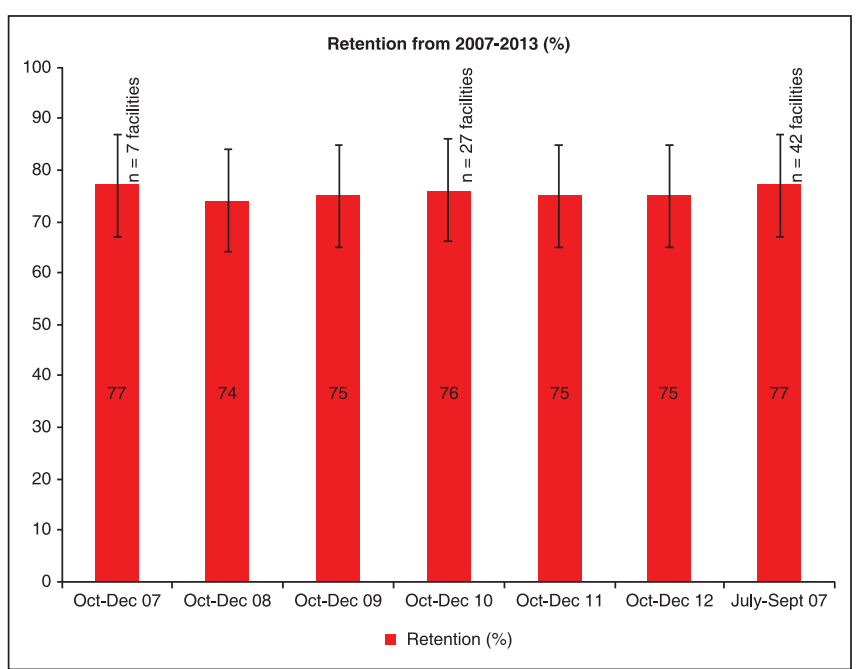

Figure 4: Five-year (2007-2013) patient retention in care

multi-disciplinary teams, site-specific challenges are shared and solved collectively, sometimes leading to the modification of standard operating procedures addressing local issues at participating sites.

Facility staff was receptive to community representatives, which enabled the partnership dimension of the clinic and the community to be realized. This initiative showed the significance of health facility leadership, signaling their willingness to hear the voice of the community through the CD4 ambassadors. Such openness also contributed to the motivation of community representatives to serve on some of the hospital quality improvement committees. Where community representatives led the health education sessions, the sessions focused on issues that the communities felt were important. Further, fulfilling these roles served a capacity-building function, community representatives gained skills and confidence in interacting with the facility teams and in exercising leadership. In all the facilities, the hospital management teams gave official recognition to the community representatives, and were strongly supportive of the committees. In the regional hospital financial support was incorporated.

The role of networks in encouraging disclosure and improving community perceptions of people living with HIV, hence reducing stigma, suggests that individual and collective empowerment is a significant product of an effective network. Connected with this is the capacity for improving self-perception, and indeed hope, transitioning from a state close to despair, to one characterized by motivation to survive and share with others that it is possible to live with HIV. The number of active support groups in the region grew considerably and the buddy system was helpful in seeking social support and decreasing stigma in the region. Meaningful engagement of people living with HIV can contribute to interventions to mitigate HIV stigma. ART and group-based approaches are opening up new avenues for the collective participation of people living with HIV to change community attitudes towards HIV. Current approaches to reducing stigma should be extended beyond interventions that seek to increase the resilience and coping mechanisms of individuals, 
to those that build the capacity of groups to collectively challenge stigma. Our findings build and expand on the conceptual framework of effective approaches for reducing HIV stigma by Brown et al. (2003). ${ }^{[31]}$ This framework suggests that a high level of interaction and proximity between people with HIV and their communities demystifies HIV and reduces stigma. ${ }^{[32]}$ Although support groups of people living with HIV have been known to exist elsewhere, ${ }^{[33]}$ what was different about the groups in this study was how they were meaningfully involved not just in receiving, but also in providing HIV services, ${ }^{[34]}$ and in the intervention section of this paper. This provided them greater visibility and opportunities to interact with their communities, and empowered them to educate their communities and change their stigmatizing values. In that sense, they became agents of social change. ${ }^{[35]}$

Outcomes included improved flow of patients through the clinic. Regular meetings between care and treatment health care providers with laboratory personnel improved communication and teamwork. Patients became more knowledgeable about their care, and the introduction of a buddy system served to reinforce patient involvement in their own care. Small-scale ethnographic studies suggest that HIV-positive individuals find it easier to disclose to other HIV-positive persons in support groups or in clinics than to their partners. ${ }^{[36,37]}$

Although care and treatment programs in resource-limited settings have reached millions of $\mathrm{HIV}$-infected patients, retention in care is a critical, but challenging aspect of efforts to optimize patient outcomes. For patients on ART, retention in care is needed to prevent medication interruptions, maintain immunologic benefits, prevent HIV resistance, and monitor the effects of therapy. For patients without indications for immediate initiation of ART, continuous monitoring is needed to prevent development of advanced disease. In addition, all patients benefit from the secondary prevention messages, counseling, and other ancillary services provided at regular medical visits. Importantly, our findings suggest that that 6 -year retention outcomes are still maintained at $>74 \%$ despite rapid scale of HIV clinics.

\section{CONCLUSION}

The quality improvement campaign to stimulate earlier treatment initiation for HIV patients was effective and yielded increased uptake of CD4 testing, treatment initiation and an improvement in patient retention.

Patients were empowered through this campaign to take a proactive role in their own care. Local use of data had an impact on overall effectiveness of care delivery. The CD4 campaign might be replicated in similar settings with consideration to the referral system and the network of support to improve adherence support programs.

This quality improvement initiative supports the use of patient empowerment as an effective strategy for improving patient outcomes. Future campaign models could explore strategies in the context of integration with other services such as TB, prevention of mother-to-child transmission, VCT, and improved use of data. The campaign is a pathway to collective participation of people living with HIV to challenge HIV stigma and act as agents of social change.

\section{ACKNOWLEDGMENTS}

This work was made possible through grants from the US Office of Global AIDS Coordinator under the President's Emergency Plan for AIDS Relief in resource-constrained settings and with the US Health Resources and Services Administration and the Centers for Disease Control. Our appreciation goes to Dr. Robert Redfield for his vision and stewardship for the program, the entire AIDSRELIEF team, specifically the University of Maryland, School of Medicine - Institute of Human Virology staff for their dedication to the program. We thank the patients and staff of clinics in Tanzania for sharing their continued experience in living with HIV/AIDS.

\section{REFERENCES}

1. Braitstein $P$, Brinkhof MW, Dabis F, Schechter M, Boulle A, Miotti $P$, et al. Mortality of HIV-1-infected patients in the first year of antiretroviral therapy: Comparison between low-income and high-income countries. Lancet 2006;367:817-24.

2. Bisson GP, Gross R, Strom JB, Rollins C, Bellamy S, Weinstein R, et al. Diagnostic accuracy of CD4 cell count increase for virologic response after initiating highly active antiretroviral therapy. AIDS 2006;20:1613-9.

3. Bisson GP, Gaolathe T, Gross R, Rollins C, Bellamy S, Mogorosi M, et al. Overestimates of survival after HAART: Implications for global scale-up efforts. PLoS One 2008;3:e1725.

4. Boulle A, Van Cutsem G, Hilderbrand K, Cragg C, Abrahams M, Mathee S, et al. Seven-year experience of a primary care antiretroviral treatment programme in Khayelitsha, South Africa. AIDS 2010;24:563-72.

5. Calmy A, Pinoges L, Szumilin E, Zachariah R, Ford N, Ferradini L, et al. Generic fixed-dose combination antiretroviral treatment in resource-poor settings: Multicentric observational cohort. AIDS 2006;20:1163-9.

6. Coetzee D, Hildebrand K, Boulle A, Maartens G, Louis F, Labatala V, et al. Outcomes after two years of providing antiretroviral treatment in Khayelitsha, South Africa. AIDS 2004;18:887-95.

7. Egger M, May M, Chêne G, Phillips AN, Ledergerber B, Dabis F, et al. Prognosis of HIV-1-infected patients starting highly active antiretroviral therapy: A collaborative analysis of prospective studies. Lancet 2002;360:119-29.

8. Mocroft A, Vella S, Benfield TL, Chiesi A, Miller V, Gargalianos P, et al. Changing patterns of mortality across Europe in patients infected with HIV-1. EuroSIDA Study Group. Lancet 1998;352:1725-30.

9. Zolopa A, Andersen J, Powderly W, Sanchez A, Sanne I, Suckow C, et al. Early antiretroviral therapy reduces AIDS progression/death in individuals with acute opportunistic infections: A multicenter randomized strategy trial. PLoS One 2009;4:e5575.

10. UNAIDS. AIDS epidemic. Available from: http://www.data.unaids.org/ pub/Report/2009/2009_epidemic_update_en.pdf. [Last updated on 2014 June; last cited on 2012 Sep 28].

11. Zachariah R, Ford N, Philips M, Lynch S, Massaquoi M, Janssens V, et al. Task shifting in HIVIAIDS: Opportunities, challenges and proposed actions for sub-Saharan Africa. Trans R Soc Trop Med Hyg 2009; 103:549-58.

12. Asamoah-Odei E, Garcia Calleja JM, Boerma JT. HIV prevalence and trends in sub-Saharan Africa: No decline and large subregional differences. Lancet 2004;364:35-40.

13. Egger M, Boulle A. Population effect of scaling up ART in resource-poor settings. Lancet 2008;371:1558-9.

14. Gilks CF, Crowley S, Ekpini R, Gove S, Perriens J, Souteyrand Y, et al. The WHO public-health approach to antiretroviral treatment against HIV in resource-limited settings. Lancet 2006;368:505-10. 
15. Granich RM, Gilks CF, Dye C, De Cock KM, Williams BG. Universal voluntary HIV testing with immediate antiretroviral therapy as a strategy for elimination of HIV transmission: A mathematical model. Lancet 2009;373:48-57.

16. Zachariah R, Harries AD, Philips M, Arnould L, Sabapathy K, O'Brien DP, et al. Antiretroviral therapy for HIV prevention: Many concerns and challenges, but are there ways forward in sub-Saharan Africa? Trans $\mathrm{R}$ Soc Trop Med Hyg 2010;104:387-91.

17. Boyer S, Eboko F, Camara M, Abé C, Nguini ME, Koulla-Shiro S, et al. Scaling up access to antiretroviral treatment for HIV infection: The impact of decentralization of healthcare delivery in Cameroon. AIDS 2010;24 Suppl 1:S5-15.

18. World Health Organisation. Available from: http://www.who.int/3by5/en/ [Last accessed on 2014 June].

19. PEPFAR Scientific Advisory Board $3^{\text {rd }}$ Meeting, Oct. $2^{\text {nd }}-3^{\text {rd }} 2012$, USA.

20. Pfeiffer J, Montoya P, Baptista AJ, Karagianis M, Pugas Mde M, Micek M, et al. Integration of HIVIAIDS services into African primary health care: Lessons learned for health system strengthening in Mozambique - A case study. J Int AIDS Soc 2010;13:3.

21. Sherer R, Stieglitz K, Narra J, Jasek J, Green L, Moore B, et al. HIV multidisciplinary teams work: Support services improve access to and retention in HIV primary care. AIDS Care 2002;14 Suppl 1:S31-44

22. Kumarasamy N, Flanigan TP, Mahajan AP, Carpenter CC, Mayer KH, Solomon S. Monitoring HIV treatment in the developing world. Lancet Infect Dis 2002;2:656-7.

23. Jourdain G, Le Cœur S, Ngo-Giang-Huong N, Traisathit P, Cressey TR, Fregonese $\mathrm{F}$, et al. Switching HIV treatment in adults based on CD4 count versus viral load monitoring: A randomized, non-inferiority trial in Thailand. PLoS Med 2013;10:e1001494.

24. US Department of Health and Human Services Panel on Antiretroviral Guidelines for Adults and Adolescents. Guidelines for the Use of Antiretroviral Agents in HIV-1-Infected Adults and Adolescents. Washington, DC: Department of Health and Human Services; 2011. p. 1-167.

25. World Health Organization. Consolidated Guidelines on the Use of Antiretroviral Drugs for Treating and Preventing HIV Infection. Recommendations for a Public Health Approach. Geneva: WHO; 2013.

26. Koenig SP, Kuritzkes DR, Hirsch MS, Léandre F, Mukherjee JS, Farmer PE, et al. Monitoring HIV treatment in developing countries. BMJ 2006;332:602-4.

27. Kent DM, McGrath D, loannidis JP, Bennish ML. Suitable monitoring approaches to antiretroviral therapy in resource-poor settings: Setting the research agenda. Clin Infect Dis 2003;37:S13-24.

28. DART Trial Team, Mugyenyi P, Walker AS, Hakim J, Munderi P, Gibb DM, et al. Routine versus clinically driven laboratory monitoring of HIV antiretroviral therapy in Africa (DART): A randomised non-inferiority trial. Lancet 2010;375:123-31.

29. Sawe FK, Mclntyre JA. Monitoring HIV antiretroviral therapy in resource-limited settings: Time to avoid costly outcomes. Clin Infect Dis 2009;49:463-5.

30. Peter TF, Shimada Y, Freeman RR, Ncube BN, Khine AA, Murtagh MM. The need for standardization in laboratory networks. Am J Clin Pathol 2009;131:867-74

31. Brown L, Macintyre K, Trujillo L. Interventions to reduce HIVIAIDS stigma: What have we learned? AIDS Educ Prev 2003;15:49-69.

32. Hardon A, Gomez GB, Vernooij E, Desclaux A, Wanyenze RK, $\mathrm{Ky}$-Zerbo $\mathrm{O}$, et al. Do support groups members disclose less to their partners? The dynamics of HIV disclosure in four African countries. BMC Public Health 2013;13:589.

33. Kim YM, Kalibala S, Neema S, Lukwago J, Weiss DC. Meaningful involvement of people living with HIVIAIDS in Uganda through linkages between network groups and health facilities: An evaluation study. Psychol Health Med 2012;17:213-22.

34. Parker R, Aggleton P. HIV and AIDS-related stigma and discrimination: A conceptual framework and implications for action. Soc Sci Med 2003;57:13-24.

35. Kyakuwa M, Hardon A, Goldstein Z. "The Adopted Children of ART": Expert clients and role tensions in ART provision in Uganda. Med Anthropol 2012;31:149-61.

36. Mfecane S. Narratives of HIV disclosure and masculinity in a South African village. Cult Health Sex 2012;14 Suppl 1:S109-21.

37. Dapaah J. HIVIAIDS Treatment in Two Ghanaian Hospitals: Experiences of Patients, Nurses and Doctors. Leiden: African Studies Centre; 2012.

How to cite this article: Memiah $P$, Shumba $C$, Henley $Y$ Mwakyusa S, Maghimbi A, Komba P, et al. "Know your CD4 campaign": 6-year outcomes from a quality improvement initiative to promote earlier initiation of antiretroviral therapy in Tanzania. Int J Med Public Health 2014;4:194-9.

Source of Support: Nil, Conflict of Interest: None declared. 